\title{
Criminologie
}

\section{Les problèmes éthiques, sociaux et légaux de la psychiatrie}

\section{Alice Parizeau}

Volume 15, numéro 2, 1982

Criminels et psychiatrie

URI : https://id.erudit.org/iderudit/017165ar

DOI : https://doi.org/10.7202/017165ar

Aller au sommaire du numéro

Éditeur(s)

Les Presses de l'Université de Montréal

ISSN

0316-0041 (imprimé)

1492-1367 (numérique)

Découvrir la revue

Citer ce compte rendu

Parizeau, A. (1982). Compte rendu de [Les problèmes éthiques, sociaux et légaux de la psychiatrie]. Criminologie, 15(2), 123-129.

https://doi.org/10.7202/017165ar d'utilisation que vous pouvez consulter en ligne.

https://apropos.erudit.org/fr/usagers/politique-dutilisation/ 


\section{LES PROBLÈMES ÉTHIQUES, SOCIAUX ET LÉGAUX DE LA PSYCHIATRIE * \\ Alice Parizeau}

Il est très malaisé de faire la recension d'un volume à la rédaction duquel ont collaboré trente-huit spécialistes. Nous allons donc nous contenter d'un commentaire puisqu'une analyse rigoureuse de chacun des chapitres dépasse nos compétences et la place dont nous disposons ici.

En premier lieu, il s'agit d'un ouvrage sur la psychiatrie clinique qui a le grand mérite d'être lisible, facile d'accès et à jour. En d'autres termes, bien que ce manuel comporte plus de neuf cent pages, le découpage thématique selon les chapitres, une unification du style et la présentation, où un effort a été fait pour rejoindre plusieurs types de lecteurs, permettent de le proposer non seulement aux spécialistes, ou aux étudiants. mais également aux profanes. Ajoutons que le livre est édité avec un souci évident du détail et qu'on y trouve aisément les renseignements particuliers qu'on se propose d'examiner.

Il convient de constater, par ailleurs, que les deux médecins, Pierre Lalonde et Frédéric Grunberg, sous la direction desquels cet ouvrage a été conçu et réalisé, ont réussi à lui assurer une homogénéité qu'on ne rencontre que rarement dans les ouvres scientifiques rédigées en collaboration.

\section{Les problèmes organisationnels du traitement}

Le premier chapitre porte sur une double approche, soit sur l'état actuel de la psychiatrie et sur les problèmes organisationnels, plus sociaux que médicaux à plusieurs égards. On y trouvera un relevé des troubles mentaux et des comportements qui les accompagnent qui, sans être exhaustif, permet de comprendre certains phénomènes généralement cachés à la faveur d'une épistémologie à ce point complexe que seuls les spécialistes peuvent la décoder.

En deuxième lieu, le docteur Grunberg essaie de répondre à la question : que fait-on pour aider et traiter les malades mentaux ? Certes, il ne s'agit pas là d'une critique du système de

* Psychiatrie clinique: approche contemporaine, Pierre Lalonde et Frédéric Grunberg (en collaboration), Gaëtan Morin, éditeur, Chicoutimi, 1980, Québec. 
"désinstitutionnalisation ", mais plutôt d'une explication simple permettant de comprendre en quoi cela consiste.

Tout un réseau de services partiels tels que centres de jour, centres de nuit, foyers de transition, pavillons, ateliers protégés, etc., ont été développés pour remplir cette fonction qui avait été assurée auparavant par l'hôpital psychiatrique totalitaire, écrit le docteur Grunberg.

On peut reprocher à l'auteur de ne pas préciser que ce réseau est constamment remis en question, autant au Québec qu'ailleurs, comme insuffisant, ou encore comme ne tenant pas compte des dangers réels de victimisation que peuvent présenter pour la collectivité, les malades mentaux. On peut également prétendre que la section concernant l'insertion sociale du malade psychiatrique comporte une conclusion trop pessimiste quand l'auteur écrit que :

Dans un bon nombre d'instances, le processus de désinstitutionnalisation du malade psychiatrique ne s'est soldé que par le transfert du malade de l'hôpital à un "ghetto psychiatrique ", implanté dans la communauté sans plus de liens réels avec la société ambiante pue les "salles de l'arrière" de l'asile.

Il n'en reste pas moins que ces conclusions, l'expérience pratique aidant, s'avèrent on ne peut plus vraies dans beaucoup de cas et qu'il est plus positif de le préciser, que d'éviter de regarder certaines réalités en face. La même approche, très claire, prévaut en ce qui a trait à l'examen des problèmes éthiques de la psychiatrie. Là encore, le docteur Grunberg ne discute pas, mais constate et tranche du même coup plusieurs débats qui sont à la mode dans notre milieu.

Selon lui, «le psychiatre, plus que tout autre médecin, doit faire face au dilemme moral d'avoir aussi bien à préserver l'intérêt de son malade que celui de la société ». De là, prétendre, comme le fait entre autres Thomas Szasz que "la psychiatrie dans la société moderne est devenue un instrument d'agression et de contrôle social pour imposer le conformisme tout comme l'Inquisition imposait le conformisme à partir du XII e siècle ", il n'y a qu'un pas. Mais, face à cette approche, le docteur Grunberg prend position en constatant qu'il « est heureux que la pensée de Szasz, définitivement démagogique, a eu très peu d'effet sur le plan pratique au Québec et au Canada $"$.

Parallèlement, toutefois, l'auteur de ce chapitre écrit : "Il ne fait aucun doute que l'antipsychiatrie, malgré ces écarts de langage et sa démagogie, a permis une prise de conscience salutaire de la psychiatrie, des limites de son pouvoir et de ses applications. " 
Voilà donc une entrée en matière qui a le grand mérite de condenser des concepts, des définitions et des courants d'opinion, que les autres auteurs reprennent plus loin dans les divers chapitres s'y rapportant, en termes descriptifs et explicatifs.

C'est ainsi qu'on y traite des syndromes cliniques psychiatriques, comme des troubles de la personnalité, du stress situationnel comme de l'arriération mentale. Nous l'avons mentionné plus haut; il est impossible de résumer ici un ouvrage de telle importance et nous ne tenons pas à nous limiter à une sorte de relevé des titres de chapitres et des noms de leurs auteurs. Par conséquent, nous sommes forcés en quelque sorte de ne retenir ici qu'une partie d'opinions ou des constatations pouvant se rattacher au thème fondamental de nos propres préoccupations, soit le traitement du malade mental et la protection de la société pour laquelle il peut présenter, dans certains cas, une menace réelle ou appréhendée.

\section{Certaines maladies sociales}

Sous ce terme de "menace", on peut comprendre toutefois plusieurs phénomènes. C'est ainsi qu'un alcoolique ou un toxicomane est souvent une menace pour son entourage et pour sa famille, puisqu'il provoque, ou précipite sa désintégration. Comme l'écrivent Paul-André Marquis et Jules Lambert, auteurs du chapitre 7 de l'ouvrage :

L'alcoolisme et les autres toxicomanies constituent indéniablement un problème majeur de santé publique en même temps qu'ils comportent des implications sociales fort sérieuses, d'où l'importance attribuée, ces dernières années, à ces troubles dans les récents traités de psychiatrie qui y consacrent généralement un ou deux chapitres complets.

On trouvera ensuite dans cette partie de l'ouvrage, non seulement une évaluation médicale du phénomène, mais également de son incidence dans notre milieu, ce qui permet à celui qui est intéressé à le comprendre, d'élargir son cadre de connaissances. Soulignons que les auteurs ne tiennent nullement à traiter des aspects idéologiques, si on peut s'exprimer ainsi, des toxicomanies. Tout au plus, ils constatent l'existence des liens qu'on établit à l'occasion entre le "phénomène drogue " et celui de " contre-culturel ", pour se consacrer bien davantage à la description des conséquences de l'usage des stupéfiants, qu'aux idéologies au nom desquelles on a essayé de le justifier. 
Il est important de souligner à cet égard que, de l'avis des médecins, contrairement à ce qui a été prôné dans certains milieux, "les alcooliques et les autres toxicomanes... sont confrontés... avec la diminution de leur potentiel et de leur créativité ».

Nous sommes bien loin des opinions selon lesquelles les toxicomanes, en particulier, peuvent avoir une meilleure vision des couleurs, par exemple, susceptibles de faciliter la création dans les domaines tels que la peinture, ou encore la photographie. Bien au contraire, nous constatons que les toxicomanes peuvent avoir des personnalités antisociales.

En traitant au chapitre 8 de la personnalité antisociale, Pierre Lefebvre et Jocelyn Aubut écrivent à ce propos :

Appelée aussi bien psychopathique, ou sociopathique, selon les auteurs, la personnalité antisociale est marquée par une absence apparente de contraintes morales, par l'inexistence de tout sentiment de culpabilité, et par des conduites dont le but est la satisfaction immédiate des désirs et des pulsions... L'antisocial n'entretient de rapport avec les autres que superficiels, occasionnels, utilitaires. Il est incapable d'un rapport affectif profond, sain, altruiste.

Certes, en bonne analyste sociologique, on peut objecter à cela que tout est relatif, qu'il n'est pas prouvé que tout altruiste est forcément un être sain, qu'il peut s'agir également là, d'un être antisocial, à sa manière. Le grand mérite de l'ouvrage, c'est qu'il présente des faits et des constatations de base, pouvant être, il va sans dire, commentés, critiqués ou analysés selon les tenants de diverses théories, ou courants de pensée.

La psychiatrie communautaire au Québec et ses perspectives d'avenir

Une autre dimension de ce livre qui s'inscrit dans le cadre de nos préoccupations concerne les sections et chapitres qui portent sur les aspects administratifs et légaux de la psychiatrie. Le lecteur aura l'heureuse surprise en les lisant d'apprendre des détails relatifs à l'évolution de la psychiatrie non seulement en France ou aux États-Unis, mais aussi au Québec, ce qui comme chacun sait est infiniment plus rare chez nous.

C'est ainsi que les docteurs G. Aird et A. Amyot écrivent :

Rares sont les pays qui, à l'instar du Québec, se sont dotés d'une politique de soins et santé mentale pour l'ensemble de leur territoire, et qui ont réussi, en moins de 20 ans, à réaliser une opération d'une telle envergure. Depuis 1960, la psychia- 
trie québécoise a connu et continue de connaître un dynamisme remarquable. (p. 871).

Ils abordent ensuite une rétrospective qui commence en 1960 , mentionnent l'élément déclencheur de l'ouvrage de Jean-Charles Pagé : Les fous crient au secours et retracent les étapes de l'action du docteur Camille Laurin, directeur du Département de psychiatrie, à l'époque, à la Faculté de médecine de l'Université de Montréal.

Camille Laurin avait alors constaté que :

Le remède à l'encombrement des hôpitaux psychiatriques n'est pas l'augmentation du nombre des lits, qui ne ferait que perpétuer et aggraver les maux que l'on déplore. La véritable solution réside dans l'instauration d'un système nouveau, qui redonne au malade sa dignité et la chance d'être traité comme il se doit.

Le gouvernement a réagi, une Commission d'étude des hôpitaux psychiatriques a été créée et les trois enquêteurs: Dominique Bédard, Denis Lazure et Charles Roberts, ont recommandé, entre autres, l'ouverture de services psychiatriques dans les hôpitaux généraux, la régionalisation des services psychiatriques et le recrutement des travailleurs de la santé spécialisés. Ce fut donc un projet d'envergure qui a été mené à bien par le docteur Dominique Bédard, puis, entre 1971 et 1977, ce fut la crise provoquée par le ministre Claude Castonguay « dont les projets de refonte des services de santé s'avèrent désastreux pour la psychiatrie » (p. 869).

Voici donc une période où les services psychiatriques se désorganisent, avec, en 1975, l'intervention du docteur Gilles Lortie qui réussit à les sectorier en obtenant, pour ce faire, la collaboration des conseils d'administration de divers hôpitaux.

Où en sommes-nous aujourd'hui ?

À cette question angoissante, les auteurs de ce chapitre répondent ce qui suit :

Le système de soins actuels est peut-être plus souple, plus mobile, moins chronicisant, mais il met également énormément de pression sur la société et sur les familles. Il laisse un grand nombre de "fous " en liberté et ne se préoccupe pas toujours assez de la qualité de vie des psychotiques chroniques qui passent leurs journées à errer dans les gares, les centres commerciaux, les quartiers des magasins, quands ils ne demeurent pas terrés dans une chambre sordide (p. 874).

Il est urgent de créer, concluent les deux médecins, des établissements spécialisés d'hébergement pour malades mentaux 
chroniques, ces établissements devant être aussi nombreux que variés afin de procurer à chaque malade le milieu de vie le plus approprié (p. 874).

Parmi ces établissements, on reconnaît aussi bien des familles d'accueil ou appartements communautaires, que des centres d'accueil, des foyers thérapeutiques ou des communes thérapeutiques. En somme, on préconise un système souple, adapté aux divers types de malades et à leurs besoins réels. Tout n'est pas parfait, mais on reconnaît l'investissement de la collectivité québécoise et les résultats qu'on a pu obtenir ainsi.

\section{La psychiatrie, le droit pénal et la responsabilité médicale}

Le trente-neuvième et dernier chapitre de l'ouvrage est consacré finalement à la psychiatrie légale. On y trouvera tout aussi bien des renseignements concernant les divers types de cure, libre ou fermée, qu'un rappel des législations et des procédures pertinentes dans les cas de l'incapacité du malade mental. Les comparaisons avec les législations des autres provinces canadiennes, ainsi qu'avec celles des États-Unis, permettent de mieux situer les concepts qui prévalent au Québec par rapport aux autres contextes culturels.

Les deux auteurs de ce chapitre, bien que médecins, F. Grunberg et G.B. Gravel, ne négligent aucune approche, y compris l'analyse de la jurisprudence, ni aucun renseignement relatif aux divers modes de traitement, aux droits du patient à donner son consentement, comme aux responsabilités du corps médical.

La conclusion est en même temps une sorte de credo :

Le psychiatre engagé dans le processus juridique, écrivent $F$. Grunberg et G.B. Gravel, ne devra jamais perdre de vue qu'il agit toujours en tant que médecin, dans les limites des connaissances des sciences médicales. Il ne devra non plus jamais perdre de vue que l'objet premier du psychiatre est de porter secours aux malades en prise avec la maladie mentale qui, quelquefois, enlève à l'individu la liberté d'agir en tant qu'être humain autonome. C'est la restauration de cette liberté humaine et individuelle, et non pas le conformisme à un ordre social et collectif, qui demeure l'objet privilégié de la psychiatrie (p. 905).

Il nous semble que cette conclusion répond à bien des questions angoissantes liées aux choix entre la protection du malade et celle de la société et qu'elle exprime fondamentalement l'avis, partagé par la plupart des membres du corps médical. Face au patient, il ne s'agit pas pour le psychiatre de justice objective, puisque la mala- 
die est déjà une injustice en soit, mais des droits du malade, dont il assume la responsabilité, en tant que médecin traitant.

\section{Conclusion}

L'éternel dilemme entre la liberté et la responsabilité des êtres qui, par définition, ne sont pas responsables de leurs actes, n'est pas, et ne sera probablement jamais résolu. Tout le domaine des maladies mentales et même des troubles de personnalité, demeure en outre dominé par des mythes qui sont d'autant plus difficiles à dédramatiser qu'ils s'accompagnent des descriptions fort anciennes, des préjugés et des craintes qui ne se raisonnent pas.

Autant il est simple de constater qu'un membre de la famille souffre d'une maladie cardiaque, rénale ou autre, autant il est particulièrement délicat d'admettre qu'il est schizophrène ou paranoïaque. On a beau accepter que le diabète peut être héréditaire et se consoler en songeant à l'insuline, quand on pénètre dans le monde mystérieux de la folie, les réactions ne sont plus les mêmes et nous en sommes tous tributaires.

C'est dans cette optique que le livre : Psychiatrie clinique : approche contemporaine, réalisé sous la direction de Pierre Lalonde et Frédéric Grunberg, doit être lu par les spécialistes, comme par les profanes. Le remarquable effort de clarté de ses auteurs lui enlève cet aspect rébarbatif qui rend beaucoup d'ouvrages scientifiques inabordables. La division des chapitres et des sections est faite de manière à ce que chacun puisse y puiser ce qui le préoccupe ou plus simplement l'intéresse. Dans son ensemble, l'ouvrage apporte un regard simple sur la maladie, le patient et le traitement, comme sur les aspects sociaux des divers phénomènes qui y sont reliés. On ne se pose plus la question si dans notre milieu, si au Québec, les méthodes utilisées sont aussi avancées qu'ailleurs, parce que les auteurs ne font à aucun moment abstraction des réalités de notre collectivité tout en les comparant au besoin à celles des autres contextes socioculturels.

Il s'agit là, en somme, d'un des rares livres scientifiques qu'il convient d'introduire partout et qui a sa place dans toutes les bibliothèques, autant comme ouvrage de référence que comme ensemble des textes permettant une réflexion plus approfondie sur des phénomènes qui ont peut-être cessé d'être des tabous, mais qui ne demeurent pas moins un sujet de préoccupations difficilement avouables au grand jour pour beaucoup d'individus et de familles. 